\title{
Nonequilibrium Temperatures Measurement in an Arc-Heated Airflow by the Optical Diagnostic Method
}

\author{
By Tetsuya YAMADA and Yoshifumi INATANI \\ Institute of Space and Astronautical Science, Sagamihara, Japan
}

(Received September 20th, 2001)

\begin{abstract}
An understanding of the aerothermophysical feature of the high-enthalpy flow is important for designing atmospheric hypersonic flight vehicles based on the ground-heating test results. Nonequilibrium temperatures of nitric oxide of the arc-heated airflow were measured by the optical diagnostic method in the Institute of Space and Astronautical Science (ISAS) arc wind tunnel facility as a part of the flow characterization. Nonequilibrium temperatures of NO $\gamma$-band $\left(A^{2} \Sigma-X^{2} \Pi_{1 / 2}\right)$ measured experimentally by 2 -line and multiline laser-induced fluorescence spectroscopy techniques exhibit separation between vibrational and rotational temperatures. The temperature relaxation along the flow has been compared with the emission spectroscopic data.
\end{abstract}

Key Words: LIF, Nonequilibrium Flow, Arc-Heated Airflow

\section{Nomenclatures}

A : Einstein's transition probability for spontaneous emission, $1 / \mathrm{sec}$

$B$ : $\quad$ Einstein transition probability for stimulated emission, $\mathrm{m}^{3} /\left(\sec ^{2} \cdot \mathrm{J}\right)$

$b:$ absorption or stimulated emission rate, $1 / \mathrm{sec}$

$c$ : speed of light in vacuum, $\mathrm{m} / \mathrm{s}$

$E_{v}, E_{r}$ : vibrational/rotational energy, $\mathrm{J}$

$F$ : observed fluorescence signal energy

$N$ : total number density of observed molecules, $1 / \mathrm{m}^{3}$

$h$ : Plank's constant, J.sec

$I_{v}: \quad$ spectral irradiance, $\mathrm{W} /\left(\mathrm{m}^{2} \cdot 1 / \mathrm{sec}\right)$

$j$ : rotational quantum number

$P$ : observed fluorescence signal power, $\mathrm{W}$

$Q:$ quenching rate, $1 / \mathrm{sec}$

$Z_{i}: \quad$ partition function of $i$ energy

$T_{r}$ : rotational temperature, $\mathrm{K}$

$T_{v}$ : vibrational temperature, $\mathrm{K}$

$V:$ sample collection volume, $\mathrm{m}^{3}$

$v:$ vibrational quantum number

$\varepsilon:$ fluorescence collection efficiency of optical device

$\Omega$ : collection solid angle, str

Superscript/subscript

'I" : value in the upper/lower state

0 : value at initial state

\section{Introduction}

The designing of a thermal protection system for reentry and entry vehicles flying through the earth's atmosphere or other planet's atmosphere, traveling either to or from space, poses severe challenges to aerothermodynamic and material

(C) 2002 The Japan Society for Aeronautical and Space Sciences research. The chief points of these challenges are the estimation of the aerodynamic heating environment and an understanding of the behavior of the thermal protection material in the flight environment. The thermochemical properties of the air in the shock layer in front of the vehicle body are dominated by flow enthalpy, surface pressure related to the body shape, and so on. These conditions in turn affect surface phenomena such as aerodynamic heating, and the recession rate of thermal protection material. A duplication of flight environments on the ground by the use of appropriate wind tunnel is desirable in the research and development of heatshield materials. Nowadays we recognize that numerical analysis is truly a strong tool, but even so, it needs to be validated by the data of other flight or ground flight environment simulators. Lacking sufficient flight experiments, the validations are currently conducted in comparison with well-characterized flight environment simulators.

The primary role of arcjet facilities in aerospace research and development has been a source of long-duration high enthalpy flow to simulate flight environments for material testing and development. At the Institute of Space and Astronautical Science (ISAS), the arc wind tunnel facility has been used for heating tests of thermal protection materials such as carbon phenolic ablator for reentry capsules ${ }^{1)}$ and insulator materials of the sounding rocket fins. ${ }^{2)}$ Although an arc wind tunnel in general does not perfectly duplicate flight environment from the standpoint of flow enthalpy, impact pressure, heat flux, and chemical composition, it still has great superiority in comparison with other high enthalpy wind tunnels as a heating test facility. It is quite important to understand the differences between a real flight environment and the test condition and its effect on the behavior of thermal protection material to reduce the weight of heatshields.

An arc-heated airflow exhibits strong chemical and thermal nonequilibrium. Although macroscopic property such 
as bulk enthalpy, heat flux can be obtained through conventional probes, the optical diagnostic technique is nonintrusive and useful in the measurements of microscopic properties such as chemical composition and nonequilibrium temperatures. Although the accessible species are limited because of available excitation wavelengths, the laser diagnostic techniques in flow research remains advantageous and offers spatially precise information versus other emission spectroscopic methods. Recent progress in laser technology and measurement techniques has succeeded in providing significant information about the characteristics of archeated airflow. It would be significant to measure the rotational and vibrational temperatures of various molecules in arc heated airflow as an index to determine the degree of nonequilibrium. Nonequilibrium temperatures are able to be measured in laser induced fluorescence (LIF) or spontaneous Raman scattering (SRS). The rotational and vibrational temperatures of $\mathrm{N}_{2}$ can be measured by $\mathrm{SRS}^{3)}$ in principle. However, it is estimated from preliminary experiments that scattering signal of $\mathrm{N}_{2}$ in an arc-heated airflow is not strong enough to conduct spectroscopic analysis because the scattering cross section is not large enough against relatively high partial pressure of $\mathrm{N}_{2}$ in the arc-heated airflow. $\mathrm{O}_{2}$ temperature measurements were conducted by LIF by the use of the Schumann-Runge band system in general for the supersonic flow ${ }^{4,5}$ but it is also estimated that $\mathrm{O}_{2}$ LIF intensity in the arc-heated airflow is too slight to be detected for the same reasons. Especially in regard to $\mathrm{O}_{2}$, Schumann-Runge (S-R) band system, which is very easy to access, is a great predissociative band with high predissociative probability. Since the fluorescence power is in inversely proportional to the predissociation probability, it is small in the S-R band. Although the partial pressure of NO is smaller than that of $\mathrm{O}_{2}$, NO LIF is thought to be strong enough. The NO $\gamma$-band $\left(A^{2} \Sigma-X^{2} \Pi_{1 / 2}\right)$ is not predissociative, and the collisional quenching is not such a severe problem in an arcjet environment. The NO temperatures were previously measured by 2-photon excitation LIF. ${ }^{6}$ ) The flow properties, which include velocity, translational temperature, and $\mathrm{N}$ concentration, were measured in $\mathrm{N}_{2}$ /argon flow by the use of two-photon excitation LIF technique. ${ }^{7)}$ Qualitative NO distribution and rotational temperatures of NO are evaluated. ${ }^{8)}$ The flow field generated in the high enthalpy facility is investigated by the use of the 2D-LIF diagnostic technique of NO. ${ }^{9}$ )

As an LIF principle for molecular systems, the two level energy model is often adapted. It is valid only for situations where rotational relaxation is either frozen or completely relaxed. Most fluorescence situations reside between these limits, and the rotational transfer needs to be accounted for.

Nonequilibrium temperatures in the electronic ground state are not guaranteed to be the same as those measured in a higher electronic state. The thermometry by LIF enables a measurement of temperatures in the electronic ground state, and the emission spectroscopy measures those in a higher electronic state. A comparison of these data would be of great interest in the same flow condition.

The purposes of this study are (1) to reconsider a methodology of LIF thermometry by taking into account the effect of rotational transfer and to establish NO LIF thermometry system for measuring arc-heated airflow; (2) to measure NO rotational and vibrational temperatures by LIF thermometry in the arc-heated airflow; and (3) to compare these data with emission spectroscopic data.

\section{Principle of LIF Thermometry}

\subsection{Laser-induced fluorescence}

Laser induced fluorescence spectroscopy (LIFS) techniques have been developed as a very powerful and the most widely used nonintrusive technique for the measurements of gas flows. This is mainly due to their high signal strength compared to other laser diagnostic methods (Rayleigh and Raman scattering), which generally makes two-dimensional measurements possible with excellent time and spatial resolution. Although all LIF methods are based on laser excitation followed by spontaneous emission of light from the particles, different methods of LIF are distinguished by the characteristics of the actual transition processes involved and the configuration of the LIF probing. In general, the fluorescence is emitted through a transition process from an upper to a lower energy level in conjunction with a spontaneous emission of a photon. Transitions within an electronic ground state involved small energy differences and shows the infrared spectrum. On the other hand, transitions between electronic states are generally associated with emission frequencies in the visible or UV region. Radiative relaxation processes with which fluorescence detection channels are concerned lie in the visible or UV region.

For molecular systems, the two level energy model is valid only for situations where rotational relaxation is either frozen, with no transfer to adjacent levels, or completely relaxed. In the latter situation, a very rapid rotational transfer establishes the Boltzmann distribution among the rotational levels in a given vibrational state and the "two" levels are then the vibrational levels containing the lumped rotational channels. Actually, most fluorescence situations reside between these limits and rotational transfer needs to be accounted for. Thus the essential characteristics of the present NO pumping and the resonant detection scheme should be represented by a rate-equation analysis of the four-level energy model illustrated in Fig. 1, in which the rates of the optical and collision processes connecting the upper and lower levels are shown. Levels $l$ (lower) and $u$ (upper) represent single rovibronic levels in the ground $\left(A^{2} \Sigma\right)$ and the excited $\left(X^{2} \Pi_{1 / 2}\right)$ electronic state that are coupled by the incident laser radiation. A molecule excited to level $u$ can relax to levels $l$; Levels $l$ and $u$ are the specific levels in the rotational channel that are optically connected by the absorption of the incident laser radiation and stimulated emission. Levels $l r$ and $u r$ represent the ensemble of rotational states in the lower and upper vibrational states, respectively. The 


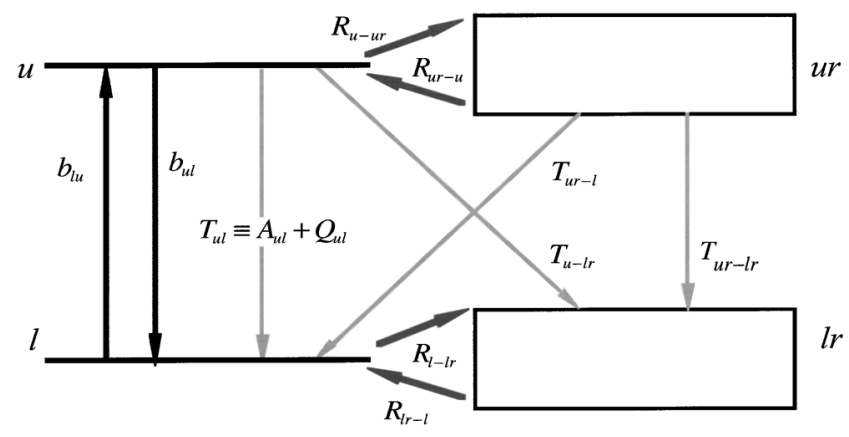

Fig. 1. Four energy level model for laser induced fluorescence spectroscopy.

downward transfer rates $T$ represent the sum of collisional quenching $Q$ and spontaneous emission $A$. The rotational transfer rates are designated by $R . b_{l u}$ and $b_{u l}$ are, respectively, the rates for absorption and stimulated emission and are related to the Einstein coefficient for stimulated emission, $B$, through the relation,

$$
b=\frac{I_{v}}{c} B
$$

where $I_{v}$ is incident laser intensity per unit frequency interval (spectral irradiance) and $B$ is the Einstein coefficient for stimulated emission, which is related to the Einstein coefficient for spontaneous emission $A$ as

$$
B=\frac{c^{3}}{8 \pi h v^{3}} A
$$

Writing rate-equations for the temporal derivatives of the rate populations, designated by $n$, as a full set of rateequations one obtains

$$
\begin{aligned}
\dot{n}_{l}= & -\left(b_{l u}+R_{l-l r}\right) n_{l}+\left(b_{u l}+T_{u l}\right) n_{u} \\
& +T_{u r-l} n_{u r}+R_{l r-l} n_{l r} \\
\dot{n}_{l r}= & +R_{l-l r} n_{l}+T_{u-l r} n_{u}+T_{u r-l r} n_{u r}-R_{l r-l} n_{l r} \\
\dot{n}_{u}= & +b_{l u} n_{l}-\left(b_{u l}+T_{u l}+T_{u-l r}+R_{u-u r}\right) n_{u} \\
& +R_{u r-u} n_{u r} \\
\dot{n}_{u r}= & +R_{u-u r} n_{u}-\left(R_{u r-u}+T_{u r-l}+T_{u r-l r}\right) n_{u r}
\end{aligned}
$$

The population conservation equation is automatically satisfied in this set and may be written

$$
n_{T}=n_{l}^{0}+n_{u}^{0}=n_{l}+n_{u}+n_{l r}+n_{u r}
$$

where the zero superscript identifies the initial level population prior to laser excitation. Adding Eqs. (3c) and (3d), we obtain under the assumption of $T_{u l}=T_{u r-l}, T_{u-l r}=T_{u r-l r}$,

$$
\dot{n}_{u}+\dot{n}_{u r}=b_{l u} n_{l}-b_{u l} n_{u}-\left(T_{u l}+T_{u-l r}\right)\left(n_{u}+n_{u r}\right)
$$

This assumption means that the rotational-rotational transition probability does not differ in rotational levels, and this is valid because only a redistribution to the vicinity of the directly excited state can be considered. As described in the previous section, the rotational transition from the directly excited state to near rotational levels are either completely frozen or relaxed.

The term for the stimulated emission from the upper state is negligible in comparison with one from the lower state: $-b_{u l} n_{u} \cong 0$. Thus,

$$
\frac{\mathrm{d}\left(n_{u}+n_{u r}\right)}{\mathrm{d} t}=b_{l u} n_{l}-\left(T_{u l}+T_{u-l r}\right)\left(n_{u}+n_{u r}\right)
$$

We assume the laser power is weak enough that the holeburning effect does not occur, and the rotational transfer is sufficiently fast that level $l$ can be maintained at its initial Boltzmann fraction $\alpha$, i.e.

$$
\frac{n_{l}}{n_{l}+n_{l r}}=\frac{n_{l}^{0}}{n_{l}^{0}+n_{l r}^{0}}=\frac{n_{l}^{0}}{n_{T}} \equiv \alpha
$$

Replacing $n_{l}=\alpha\left\{n_{T}-\left(n_{u}+n_{u r}\right)\right\}$ and integrating with the $\left(n_{u}+n_{u r}\right)=0$ initial condition, we obtain

$$
\left(n_{u}+n_{u r}\right)=\frac{\alpha b_{l u} n_{T}}{r}\{1-\exp (-r t)\}
$$

where $r=\alpha b_{l u}+T_{u l}+T_{u-l r}=\alpha b_{l u}+A_{u l}+A_{u-l r}+$ $Q_{u l}+Q_{u-l r}$ and $\left(n_{u}+n_{u r}\right)$ at $t=0$ has been assumed as the initial condition. Because population in the upper electronic state prior to laser excitation for electronic transitions in the visible and UV spectral regions is generally negligible, the initial condition is zero. In most cases for the strong UV pulse lasers, the assumption

$$
r t \gg 1
$$

can be made, and the upper level population builds up instantaneously and reaches a steady state value of

$$
\left(n_{u}+n_{u r}\right)=\frac{\alpha b_{l u} n_{T}}{r}=\frac{b_{l u}}{r} n_{l}^{0}
$$

In most situations in the arcjet environment the steady state is determined by stimulated emission rate depending on the laser intensity. The assumption of the steady state laser excitation will be shown in the following section.

The fluorescence power, $F$, emitted from an observation volume of $V$ through an observation solid angle of $\Omega$ is proportional to $n_{u} A$ and is expressed as

$$
\begin{aligned}
F & =h\left(n_{u} v_{u l} A_{u l}+n_{u r} v_{u r-l} A_{u r-l}\right) \frac{\Omega}{4 \pi} V \varepsilon \\
& \cong h v_{u l} A_{u l}\left(n_{u}+n_{u r}\right) \frac{\Omega}{4 \pi} V \varepsilon
\end{aligned}
$$

In this equation, the frequency difference between $v_{u l}$ and $v_{u r-l}$ is so small that it is not distinguished by spectrometers with a normal resolution of about $0.1 \mathrm{~nm}$. The last factor $\varepsilon$, the detection efficiency in Eq. (11), is necessary to correct for losses in the collection of the photons. The detection efficiency is determined by the optical arrangement and includes losses at the various elements in the optical path of collection, which can be assumed to be constant within the range of wavelength in the present experiment.

By inserting Eq. (10) into Eq. (11), we obtain,

$$
F=h v_{u l} A_{u l} \frac{\Omega}{4 \pi} V \varepsilon \frac{b_{l u}}{r} n_{l}^{0}
$$


Thus the fluorescence power is proportional to the number density of the initial state. Since the population is leaking from the directly pumped level, as shown in Eq. (11), the fluorescence signal is maximized by observing fluorescence from the entire upper level populations rather than by observing fluorescence from only the directly excited state. In most experiments performed in the linear fluorescence regime, the spectrometers are opened wide to achieve a larger spectral detection bandpass which maximized the fluorescence signal and thus the species detectivity. In reality, several transitions occur, and the fluorescence signal is obtained as a fluorescence superposition of transitions in the vicinity of the directly excited state.

\subsection{LIF thermometry}

Under the assumption that the Boltzmann distribution can be respectively applied to the vibrational and rotational energy distribution, the initial number density of the diatomic molecules in $l$-state is expressed as

$$
n_{l}^{0}=N \frac{2 j^{\prime \prime}+1}{Z_{v} Z_{r}} \exp \left[\frac{-E_{v}}{k T_{v}}+\frac{-E_{r}}{k T_{r}}\right]
$$

where $N$ is the total number density of the target molecule and $Z_{v}$ and $Z_{r}$ the vibrational and rotational partition function, respectively. $E_{v}$ and $E_{r}$ denote the vibrational and rotational energies, respectively, and are expressed as

$$
\begin{array}{r}
E_{v}=h c\left[\omega_{e}\left(v+\frac{1}{2}\right)-\omega_{e} x_{e}\left(v+\frac{1}{2}\right)^{2}\right. \\
\left.+\omega_{e} y_{e}\left(v+\frac{1}{2}\right)^{3}+\cdots\right] \\
E_{v}=h c\left[B_{v} j(j+1)-D_{v} j^{2}(j+1)^{2} \cdots\right]
\end{array}
$$

where $B_{v}$ and $D_{v}$ are expressed as

$$
\begin{aligned}
& B_{v}=B_{e}-\alpha_{e}\left(v+\frac{1}{2}\right)+\cdots \\
& D_{v}=D_{e}-\beta_{e}\left(v+\frac{1}{2}\right)+\cdots
\end{aligned}
$$

The coefficient included in Eqs. (15a) through (15c) are obtained from the literature. ${ }^{10)}$

Combining Eqs. (11) through (13), we obtain

$$
F=h v_{u l} \frac{\Omega}{4 \pi} V \frac{b_{l u}}{r} N \frac{2 j^{\prime \prime}+1}{Z_{v} Z_{r}} \exp \left[\frac{-E_{v}}{k T_{v}}+\frac{-E_{r}}{k T_{r}}\right]
$$

The excitation method requires light sources that are tunable, and they must exhibit very narrow bandwidths to avoid simultaneous excitation of several rotational levels in the lower laser-coupled states. If the light source can be tuned to stimulated electronic transitions from different single rotational levels within a vibrational level, the observed fluorescence intensity can be obtained from Eq. (16). The transition of molecules from the upper electronic level to the ground level is not always connected with the emission of light, since the collisional quenching exists.
Although the target molecule is nitric oxide in the present study, the following thermometry can be generally applied to other diatomic molecules. If LIF measurements are to be performed, the structure and distribution of the energy levels of the species of interest along with possible transitions and transition probabilities must be known in advance. Temperature measurements are possible by two methods; One is a method to excite molecules by scanning stimulated transitions and observing the total emissions per fixed excitation wavelength. The other is to observe the spectral distribution of the emissions induced by the absorption center wavelength fixed to a given transition. The experimental scanning through the spectral range of transitions of the target species usually takes much time, though the resolution is excellent up to the laser line width. Although it is suitable for measurement in an arc wind tunnel facility from the stand point of operation time to use two or several distinct lines for excitation with fixing the excitation wavelength at the center of the absorption and to compare the broadband fluorescence signals.

\subsection{Vibrational temperature measurement}

The vibrational temperature can be measured by 2-line LIF. ${ }^{11)}$ The basic idea of 2-line LIF is to measure the relative population of two states and to calculate the temperature under the assumption of Boltzmann distribution. An important feature of the technique is excitation to the same upper state. For each fluorescence transition then, the effects of quenching and rotational or vibrational energy transfer, or both are exactly the same. The fluorescence efficiency then cancels out for each transition and the ratio of the two LIF intensities correspondent to the different vibrational state with the same rotational and electronic state are expressed as follows from equations (11) and (12).

$$
\frac{F_{v^{\prime \prime} 1}}{F_{v^{\prime \prime} 2}}=\frac{v_{u l 1}}{v_{u l 2}} \frac{b_{l 1 u}}{b_{l 2}} \frac{r_{l 1}}{b_{l 2 u}} \exp \left[\frac{-\left(E_{v 1}-E_{v 2}\right)}{k T_{v}}\right]
$$

Since the same rotational states are selected for vibrational temperature measurement, $r_{l 1}$ and $r_{l 2}$ cancells each other out in Eq. (17). The two vibrational energy levels correspondent to the two excited levels are calculated on Eqs. (14) and (15). Thus the only unknown vibrational temperature can be estimated.

To conduct LIF thermometry, absorption lines must be chosen by considering laser wavelength, distribution of NO, and the selection rule of each transition. Although the excitation scheme or wavelength should be so selected that the temperature sensitivity is large, they are both restricted by the available wavelength that can be generated by the present laser system. The available excitation wavelength is around $230 \mathrm{~nm}$ because of the range of the second harmonic generator. The absorption lines that satisfy these requirements are the NO $\gamma$-band system and the target scheme is excitation from $v^{\prime \prime}=0$ (vibrational ground state) and $v^{\prime \prime}=2$. Figure 2 shows the population distribution of vibrational states in the NO $\gamma$-band $\left(A^{2} \Sigma-X^{2} \Pi_{1 / 2}\right)$ system with a parameter of vibrational temperature. In the temperature range of 1000 to 


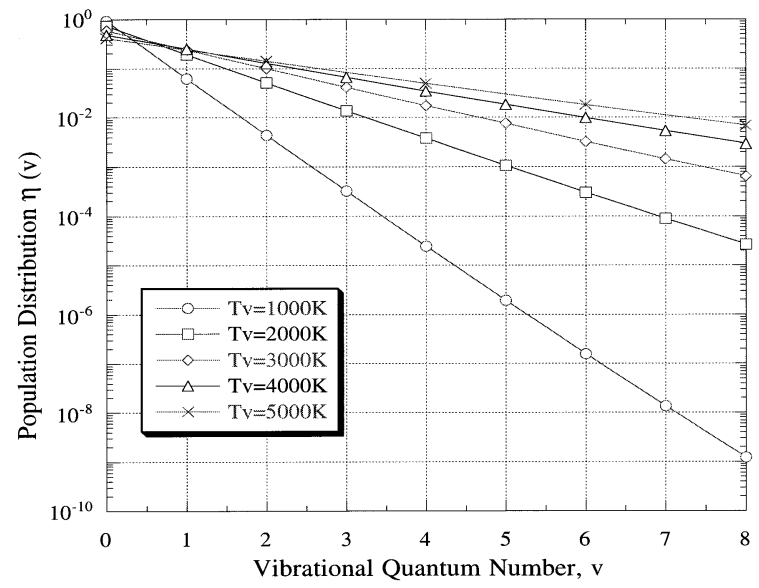

Fig. 2. Vibrational distribution of NO $\gamma$-band as a function of the vibrational temperature.

$2000 \mathrm{~K}$, the population in $(v+1)$ state is an order of magnitude smaller than that in $v$ state, which makes it difficult to excite the higher vibrational state because of a low LIF signal level. Since no selection rules are applied on the vibrational transition, excitation-to-the-same-state LIF described before can be applicable for vibrational temperature measurement.

\subsection{Rotational temperature measurement}

The situation is different for estimating rotational temperature. Figure 3 shows rotational distribution in the NO $X^{2} \Pi_{1 / 2}$ state. The rotational distribution is sensitive to the rotational temperature, and the order of population in $j^{\prime \prime}+1$ states is of almost the same order of magnitude as that in $j^{\prime \prime}$. Moreover, the intervals of excitation wavelength between $j$ number ranges 30 to $40 \mathrm{pm}$ per $j^{\prime \prime}$-number, which enables us to conduct multiline LIF thermometry. To obtain rotational temperature, excitation to the same rotational quantum number should be conducted while remaining the vibrational quantum number same. The selection rules for the rotational transition permits only $j^{\prime}=j^{\prime \prime}+0, \pm 1$ transition, which in principle makes the multiline LIF excitation with same upper state difficult. On the other hand, the as-

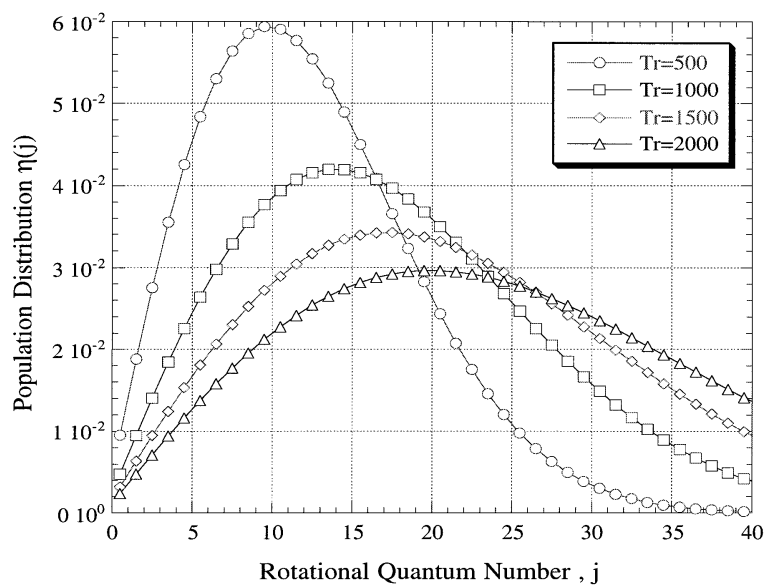

Fig. 3. Rotational distribution of $\mathrm{NO} \gamma$-band as a function of the rotational temperature.
Table 1. Absorption lines used for LIF thermometry.

\begin{tabular}{lcc}
\hline Vibrational temperature measurement & \\
\hline No. & Absorption line & $\begin{array}{c}\text { Wavelength in vacuum } \\
{[\mathrm{nm}]}\end{array}$ \\
\hline 1 & $\gamma(0,0) Q_{22}(11.5)$ & 226.464 \\
2 & $\gamma(0,2) Q_{22}(11.5)$ & 247.305 \\
\hline \multirow{2}{*}{ Rotational temperature measurement } & \\
\hline \multirow{2}{*}{ No. } & Absorption line & 226.673 \\
\hline \multirow{2}{*}{1} & $\gamma(0,0) Q_{22}(5.5)$ & 226.545 \\
2 & $\gamma(0,0) Q_{22}(9.5)$ & 226.464 \\
3 & $\gamma(0,0) Q_{22}(11.5)$ & 226.215 \\
4 & $\gamma(0,0) Q_{22}(16.5)$ & 225.896 \\
5 & $\gamma(0,0) Q_{22}(21.5)$ & 225.508 \\
\hline
\end{tabular}

sumption that the collisional quenching among different rotational number is almost of the same order and negligible is a reasonable approximation for this LIF measurement. The Hönl-London factor can be represented by that of the directly excited state by summing up all fluorescence after redistribution along the rotational states as described in the previous section. Thus multiline LIF thermometry was used for the rotational temperature measurement. Unlike the 2-line case of the vibrational thermometry, multi-line thermometry reduces measurement errors. Table 1 summarizes the absorption lines used in this experiment.

\section{Experimental Instrumentation and Procedure}

\subsection{Arc wind tunnel facility}

All experiments are conducted in the ISAS arc wind tunnel facility where the Huels-type arc heater (HARC) and the segmented-disk type arc heater (SARC) are equipped for thermal protection material testing and the study of the nonequilibrium of high-enthalpy flow. Although the enthalpy level of HARC is no more than $12 \mathrm{MJ} / \mathrm{kg}$, that of SARC ranges from 3 to $22 \mathrm{MJ} / \mathrm{kg}$ depending on the number of disk packs. In this study, SARC was operated in 1-pack configuration to generate relatively low-enthalpy airflow as

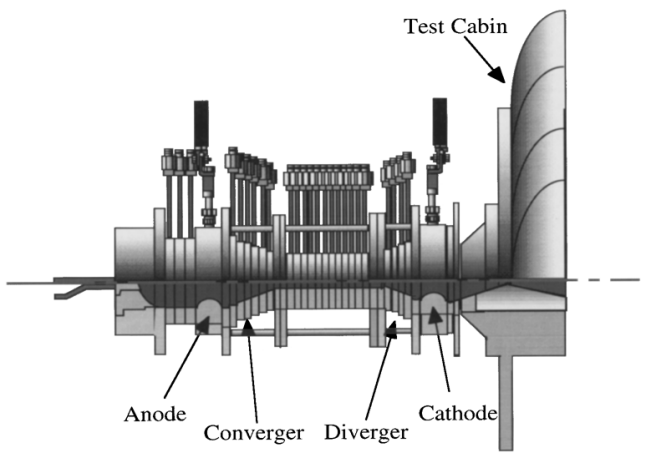

Fig. 4. ISAS segmented disk-type arc heater in 1-pack configuration. 
Table 2. Arc heater operation conditions.

\begin{tabular}{ll}
\hline Plenum pressure & $5-10.5 \mathrm{~kg} / \mathrm{cm}^{2}$ \\
Specific enthalpy & $9.5 \mathrm{MJ} / \mathrm{kg}$ \\
Ambient pressure & 1 Torr \\
\hline
\end{tabular}

shown in Fig. 4. The normal test condition in this study is summarized in Table 2. To observe temperature relaxation at a different area ratio, the nozzle with a small area ratio is used. Since the nozzle flow is under-expanded in comparison with the ambient pressure, the plume is suddenly expanded after passing through the nozzle exit with expansion fans. The LIF measurement is conducted in the free stream. For a quantitative discussion, geometrical area ratio was introduced, which is the virtual area ratio of the area determined by virtual extension of the nozzle wall at the test point to the nozzle throat area. The geometrical nozzle ratio of the measurement point ranges 16 to 100 .

\subsection{LIF system}

Figure 5 shows a schematic view of the NO-LIF temperatures measurement system. The excimer laser (LUMONICS PM-882) with $\mathrm{XeCl}$ as a laser medium generates $308 \mathrm{~nm}$ wavelength with a broad linewidth of $1 \mathrm{~nm}$. The laser beam from the excimer laser is leaded to the dye laser (LUMONICS HD-500) as a pump beam. The dye laser is tuned to the wavelength of about $452 \mathrm{~nm}$ or $494 \mathrm{~nm}$ with a linewidth of $0.04 \mathrm{~cm}^{-1}$ at $200 \mathrm{~nm}$. From the dye laser beam, BBO generates a second harmonic, which is $226 \mathrm{~nm}$ or $247 \mathrm{~nm}$. The energy and pulse width of the second harmonic are about $1 \mathrm{~mJ}$ per pulse and $20 \mathrm{nsec}$, respectively.

It takes much time to scan the whole absorption bandwidth expressed as the Voigt function by the tunable dye laser because of the low repetition rate of pumping excimer laser $(10 \mathrm{~Hz})$. A fluorescence signal for higher $\left(v^{\prime \prime}=2\right)$ vibrational level excitation is so low that signal integration is required. The laser wavelength for the excitation is then locked to the absorption center wavelength measured and checked by the use of a NO gas cell in advance to the LIF temper-

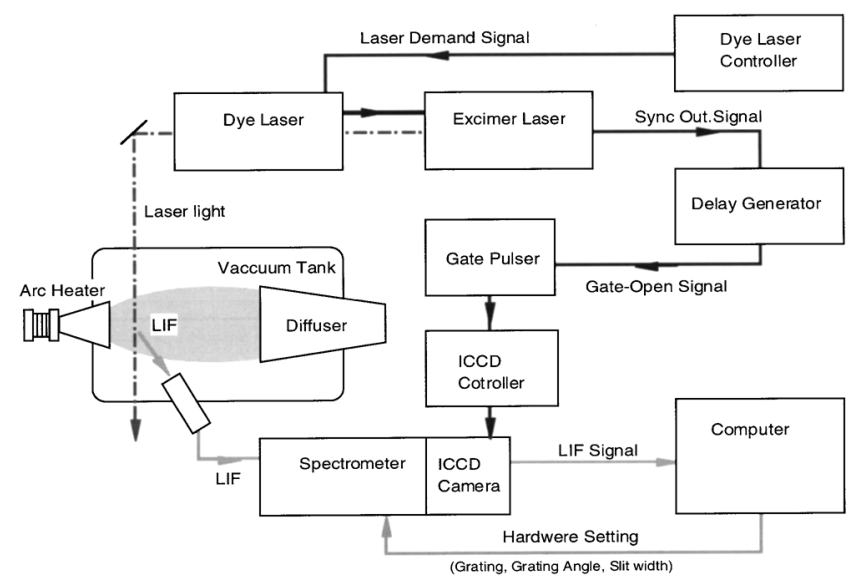

Fig. 5. LIF measurement system diagram. ature measurement. A LIF spectrum at a given excitation wavelength of the laser radiation is stored for one operation run of the arc heater.

The spectrometer system (HAMAMATSU PMA-50) composed of a Zerni-Turner type of spectrometer (HAMAMATSU C5095) and a 2-dimensional CCD array system, which enables obtaining of spectroscopic data with positional information. The fluorescence signal is collected by focusing lenses and lead to the spectrometer through linear optical fiber, which is one-dimensionally arrayed lump of small fibers and can lead light to the spectrometer while maintaining positional information. The slit width is $200 \mu \mathrm{m}$ and grating is $150 \mathrm{gr} / \mathrm{mm}$. The spectrum data are detected by ICCD camera (HAMAMATSU C6394-02) and are recorded by a personal computer (Macintosh PM-8200AV). The gate of the ICCD camera is controlled by a gate pulser (HAMAMATSU C4568) and a delay generator (STANFORD Instrument DG535). The delay generator makes use of s excimer laser synchronizer-out signal as the trigger and generates a synchronized TTL triggering signal for following the instruments. The gate-pulser is triggered by the signal and controls the gate of the image intensifier. The gate duration of this measurement is in the range of $200 \mathrm{nsec}$.

The time history of the LIF signal from the arcjet is obtained by a photo-multiplier tube. The emission spectroscopic measurement has been conducted with almost the same systems as LIF measurement, except the point that the laser system is not included.

\subsection{Experimental procedure}

The errors of the absorption-center excitation method depends on the degree of how exactly the excitation laser wavelength is identical to the target absorption center wavelength. The laser wavelength shifts because of the linear thermal expansion of the internal grating of the laser by an order of $1 \mathrm{pm}$ per $1^{\circ} \mathrm{C}$ change of the room temperature. The wavelength shift resulting from thermal expansion of the grating is a serious problem even if the room temperature is conditioned. This is because the full width of the half height of the laser absorption, which is dominated by doppler broadening, is comparable order of magnitude (about 1 to $2 \mathrm{pm}$ in $200 \mathrm{~nm}$ range). Moreover, the accuracy of the molecular constants referred to in the literature is an order of $10 \mathrm{pm}$. The pulsed wavemeter (Burleigh Model 5500) is then used to measure the laser wavelength for coarse wavelength matching. The principle technique of the wavemeter involves passing the input laser light through the two Fabry-Perot interferometers (etalons) that are calibrated against an internal He-Ne reference laser. Since the accuracy in the range of $300 \mathrm{~nm}$ is $\pm 0.2 \mathrm{~cm}^{-1}$ (about $5 \mathrm{pm}$ ), the wavemeter helps to distinguish one absorption line from the next, regardless of the uncertainty of the theoretical absorption wavelength. The absorption-center wavelength is then checked and corrected by measurement of the intensity maximum of the LIF signal from the NO gas enclosed in a glass cell set in the position to be tested. 


\section{Results and Discussion}

\subsection{Time profile of NO-LIF signal decay}

An expression of LIF in Eq. (11) is based on the assumption designated in Eq. (9). This assumption is confirmed to be reasonable by investigation of a time-history of LIF. The fluorescence power decay after laser excitation was terminated is approximately expressed as a function of time as

$$
P=\sum_{i}^{i_{\max }}\left(A_{u l i} h v_{u l i}\right) \frac{\Omega V \varepsilon}{4 \pi} \exp \left[-\left\{\sum_{i}^{i_{\max }}\left(A_{u l i}+Q_{u l i}\right)\right\} t\right]
$$

The time-history of the LIF signal has been obtained with a photomultiplier tube (HAMAMATSU Photonics Model R955), the signals of which are stored by a digital storage oscilloscope (HP-54522A) with maximum sampling rate of $2 \mathrm{GHz}$. The time-history of the LIF signal at a typical point in the expanding arcjet flow is shown in Fig. 6.

Figure 8 shows three examples of observed LIF spectrum in the excitation of $\gamma(0,0) Q_{22}(11.5)$. The obtained time history of the LIF spectrum has undertaken fitting process by using Eq. (18) assuming the amplitude and the characteristic time to be unknowns; the value $\sum_{i}^{i_{\max }}\left(A_{u l i}+Q_{u l i}\right)$ in each situation is estimated to be $7.1 \mathrm{e}+6,6.6 \mathrm{e}+6,6.1 \mathrm{e}+6[1 / \mathrm{s}]$, respectively. The assumption designated in Eq. (11) has turned out to be valid for this transition. Since the difference of $b_{l u}$ in each excitation scheme listed in Table 1 is within $30 \%$ in the same order of magnitude, the assumption designated in Eq. (11) is applicable to all the transitions in this study.

Figure 7(a) shows a typical example of the observed LIF spectrum at a given point of the laser beam path in the flow insituation of $\gamma(0,0) Q_{22}(11.5)$ excitation, as listed in Table 1. Each peak is a fluorescence signal correspondent to the transition $\left(v^{\prime}, v^{\prime \prime}\right)=(0,0),(0,1),(0,2)$, etc., which is schematically shown in Fig. 7(b). Each peak that seems to be a sole transition actually consists of a superposition of many rovibronic transition lines of the same vibrational quantum number with different rotational numbers. Since the superposition is not resolved by the present spectrometer described in the previous section, the LIF signals as a result of the rotational-rotational transfer can also be stacked as a superposition. Figure 8 shows an ideal example of the LIF spectrum calculated by the use of SPRADIAN, ${ }^{12)}$ which is correspondent to the situation in which an infinitesimally high-resolution spectrometer is used.

The fluorescence power for estimating temperatures, which are described in Eq. (11), is corrected as follows. The experimentally obtained raw LIF spectrum $F^{\prime}$ is related to the "true" spectrum $F$ as

$$
F^{\prime}=\eta(\lambda)(F+B N)
$$

where $B N$ is the background radiation noise and $\eta$ the wavelength sensitivity of the spectrometer. Although $B N$ can be reduced if the gate-open duration of the image intensifier is decreased, the excessively short gate-open duration leads to an increase of the signal-integration number. By de-
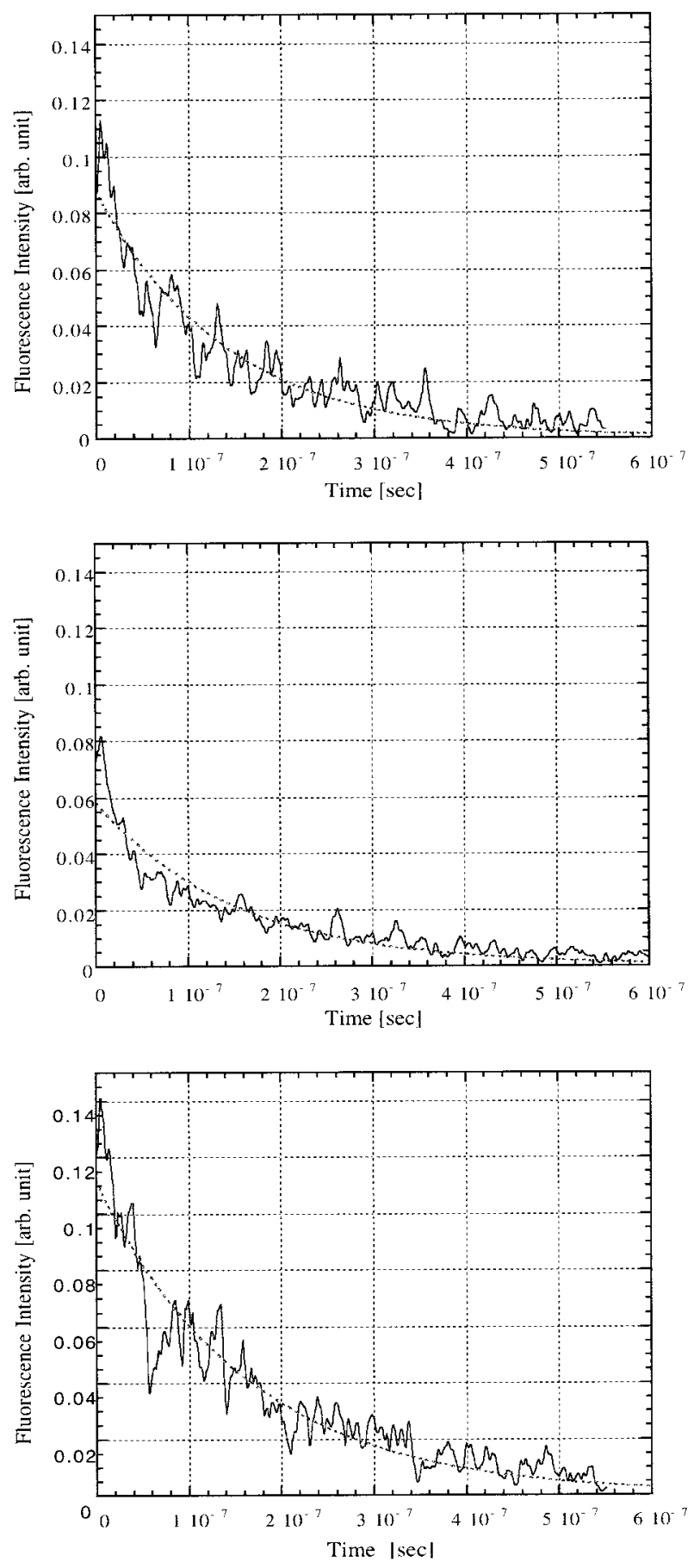

Fig. 6. Time profile of LIF decay in case of $\gamma(0,0) Q_{22}(11.5)$ excitation.

tuning the laser excitation wavelength about several tens of picometers off the absorption center, we can obtain the nonresonant signal, namely, in advance. When we subtract the background radiation noise from the raw fluorescence and correct the wavelength sensitivity, we have the corrected fluorescence spectrum.

The fluorescence energy is determined by integrating the spectrum for wavelength ranging from 200 to $350 \mathrm{~nm}$. This integration range is wide enough because more than $99 \%$ of the fluorescence energy is gathered. 

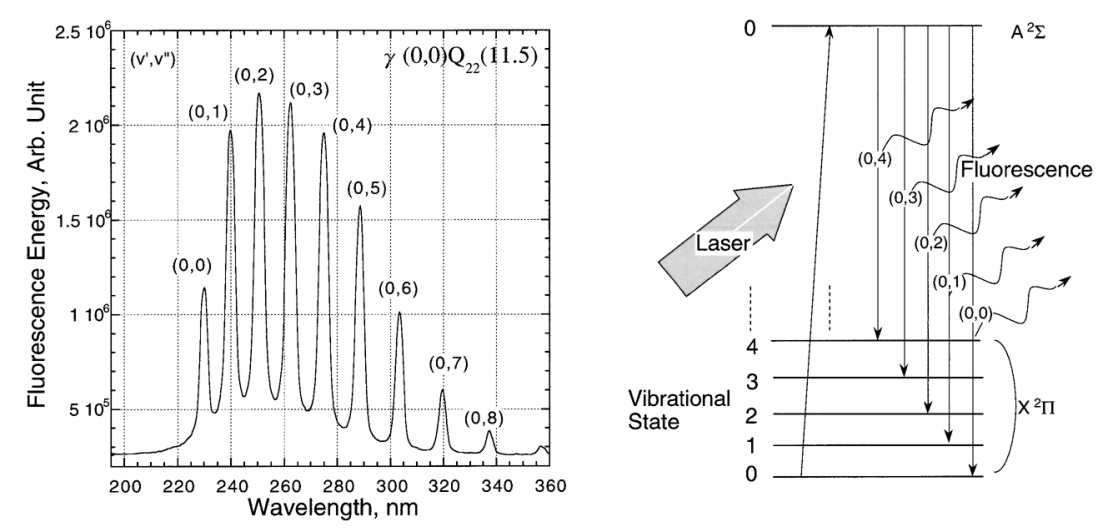

Fig. 7. Observed spectrum of NO LIF in case of $\gamma(0,0) Q_{22}(11.5)$ excitation.

\subsection{Nonequilibrium temperatures measured by LIF}

\subsubsection{Errors of temperature estimation}

Errors for temperature estimation because of the fluorescence signal intensity are generally expressed in

$$
\mathrm{d} T=\sum_{i=1}^{i_{\max }}\left(\frac{\partial T}{\partial F_{l i}} \mathrm{~d} F_{l i}\right)
$$

In regard to vibrational temperature, by substituting 2 to $i_{\max }$ and conducting differentiation in an analytical form, we obtain,

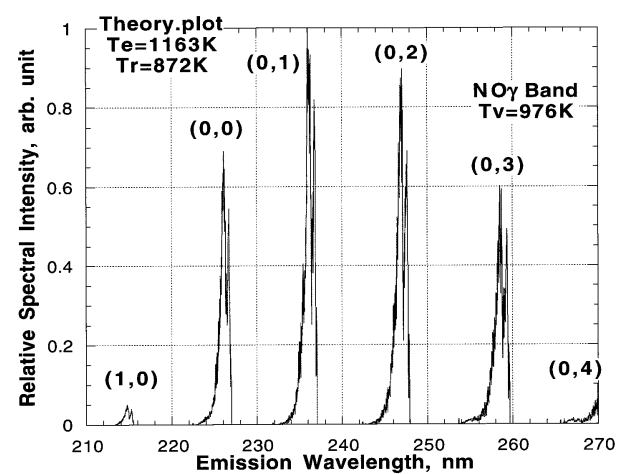

Fig. 8. Calculated spectrum of NO LIF $\gamma$-band.

$$
\mathrm{d} T=\sum_{i=1}^{2} \frac{\left[E_{v}\right]_{l 2}-\left[E_{v}\right]_{l 1}}{k}\left[\ln \left\{\frac{\left[F_{v}\right]_{l 2}}{\left[F_{v}\right]_{l 1}} \frac{\left[\frac{b_{l u}}{b_{l u}+b_{u l}}\left[1-\exp \left\{-\left(b_{l u}+b_{u l}\right) \tau_{l}\right\}\right]\right]_{l 2}}{\left[\frac{b_{l u}}{b_{l u}+b_{u l}}\left[1-\exp \left\{-\left(b_{l u}+b_{u l}\right) \tau\right\}\right]\right]_{l 1}}\right\}\right]^{-2} \frac{\mathrm{d} F}{[F]_{l i}} \equiv \operatorname{Err}(T) \cdot \frac{\mathrm{d} F}{[F]_{l i}}
$$

where the value in the largest parentheses is calculated on the constants related to the excitation scheme and the laser specification. The terms inside the natural logarithm are almost unity except the term $[F]_{u} /[F]_{l i}$, which differs by the same order of magnitude as the ratio of vibrational distribution in the selected transition scheme as a function of temperature. In this study the upper vibrational state is fixed to $v^{\prime}=0$. In the instance that one of the lower vibrational state $l 1$ is fixed to 0 , the term $\operatorname{Err}(T)$ ranges from several hundred to 2,000 for a parameter of $l 2$ and the observation temperature as shown in Fig. 9. The errors are larger in the measurements in high temperatures. The value $\mathrm{d} F /[F]_{l i}$ contains no optical collection efficiency of the system, which is already canceled at the stage of comparing relative signal intensities. Because the excitation is conducted in separate tests, the geometrical setting errors should be included. The value $\mathrm{d} F /[F]_{l i}$ is estimated to be $10 \%$ mainly as a result of the setting errors. The error of vibrational temperature is at most $\pm 200 \mathrm{~K}$ at $1000 \mathrm{~K}$ level.

The number of measurement schemes for the rotational temperature is more than that for the vibrational tempera-

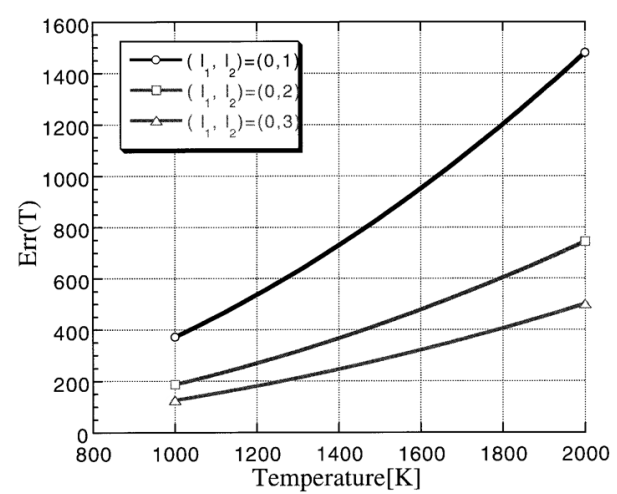

Fig. 9. Error estimation for the case of the vibrational temperature measurement.

ture, which reduces total errors despite of the same extent of fluorescence errors. Though the principle of estimating rotational temperature measurement is almost same, it cannot be analytically expressed and estimated numerically. The error of rotational temperature is estimated to be $\pm 60 \mathrm{~K}$.

The LIF intensity in the rotational excitation scheme is di- 


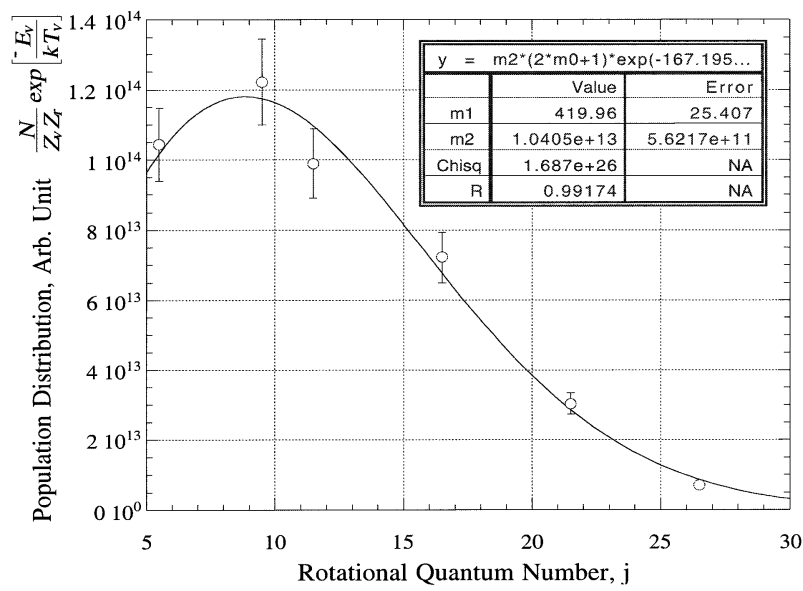

Fig. 10. Rotational distribution fitting of LIF experimentally measured.

rectly related to the rotational population distribution, which is plotted versus the rotational quantum number. This is revealed in Fig. 10, which shows the measured rotational distribution by $(0,0)$ transition at the point designated by the symbol "*" in Fig. 13. Obtained distribution agrees well with the fitting curve. Errors calculated on the data of the typical point of the laser path is adopted to all points and shown in Fig. 10. By fitting this rotational population distribution, the rotational temperature can be estimated.

\subsubsection{Radial distribution of the nonequilibrium tem- perature}

The radial distribution of nonequilibrium temperatures are measured by LIF along the linear position shown in Fig. 11. Figure 12 shows an example of the spatial distribution of the NO LIF spectrum in case of the excitation of $\gamma(0,0) Q_{22}(11.5)$. The spectrometer (PMA-50) can give a 2-dimensional image (space vs. wavelength) of the spectrum in real time. After the described procedurehas been conducted, nonequilibrium temperatures along the laser beam line A through B are estimated and shown in Fig. 13. This figure shows a separation of the vibrational temperature from the rotational temperature by $300 \mathrm{~K}$ at the center axis. The nonequilibrium in temperature has been detected by LIF thermometry. The temperatures in the nozzle center line are higher than those in boundary and the separation between rotational and vibrational temperature is about $300 \mathrm{~K}$. The

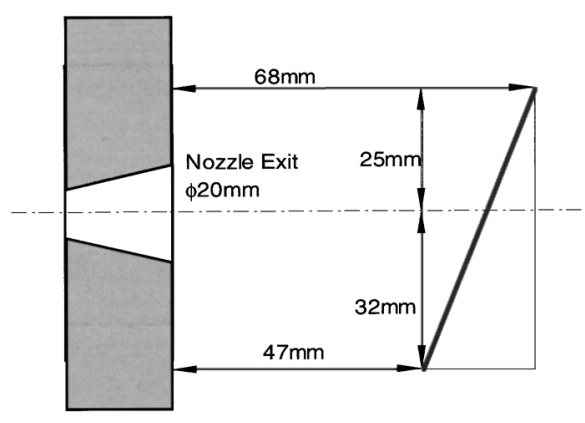

Fig. 11. Measurement line position. vibrational temperature at point $B$ is higher than that at the point A. It may reflect the relaxation process of vibrational temperature because the nozzle expansion ratio at the point $\mathrm{B}$ is slightly smaller than at the point A. Errors in the vibrational temperature is relatively large. This is caused because the fluorescence signal by $(0,2)$ transition, which enables us to know the population of $v^{\prime \prime}=2$, is very small. At future study, $(0,1)$ transition, which enables us to know the population of $v^{\prime \prime}=1$, should be apply, because the fluorescence signal of $(0,1)$ transition is expected to be much larger than that of $(0,2)$ transition.

\subsection{Temperature relaxation along flow \\ 4.3.1. Emission spectroscopy}

Besides the LIF thermometry, emission spectroscopic thermometry was also conducted. Figure 14 shows experimentally obtained emission spectrum ranging from 218 to $272 \mathrm{~nm}$ together with theoretical emission in case of the electronic temperature of $1,000 \mathrm{~K}$. The emission spectrum consists of $\gamma-, \delta$-, and $\varepsilon$-bands, respectively. The intensities of $\delta$-bands, $\varepsilon$-bands are so high that they are not emitted unless the electron temperature is up to an order of $10,000 \mathrm{~K}$. The spectrum data fitted by using SPRADIAN shows an extremely high electronic temperature up to tens of thousands of kelvin. As one reason, the collision between $\mathrm{NO}$ and $\mathrm{N}_{2}$

$$
\mathrm{NO}\left(X^{2} \Pi_{r}\right)+\mathrm{N}_{2}\left(A^{3} \Sigma_{u}^{+}\right) \rightarrow \mathrm{NO}\left(A^{3} \Sigma^{+}\right)+\mathrm{N}_{2}\left(X^{-1} \Sigma_{g}^{+}\right)
$$

is pointed out in the arc-heater flow. ${ }^{13)}$ Because of the electron-vibrational coupling, the population of the above specula electronic state becomes far from the Boltzmann distribution, which causes an incredible electronic temperature under the assumption of Boltzmann distribution. In any event, fitting in order to obtain rotational temperature requires special care in regard to the fitting range, which needs to be selected so that it contains no $\delta, \varepsilon$-bands. We wish to point out here that the vibrational and rotational temperatures of NO $\gamma$-bands obtained by emission spectroscopy are those of the electronic state band $A^{2} \Sigma$, which is different from LIF of the electronic ground state band $X^{2} \Pi_{1 / 2}$.

\subsubsection{One-dimensional nonequilibrium airflow analy- sis}

To discuss the nonequilibrium temperature distribution along the nozzle center axis obtained by LIF, emission spectroscopic data and a quasi one-dimensional numerical analysis are briefly introduced here. The quasi one-dimensional nonequilibrium flow analysis code takes into account 13 species $\left(\mathrm{O}_{2}, \mathrm{~N}_{2}, \mathrm{O}, \mathrm{N}, \mathrm{NO}, \mathrm{O}^{+}, \mathrm{N}^{+}, \mathrm{NO}^{+}, \mathrm{O}_{2}^{+}, \mathrm{N}_{2}^{+}, \mathrm{Ar}\right.$, $\left.\mathrm{Ar}^{+}, \mathrm{e}^{-}\right)$as the composition of high temperature air mixture. Reaction model used is 35 reactions, including dissociation, ionization, recombination, and charge exchange. Most are similar to Park's model and/or Dunn and Kang's. The present model adapted 5-temperature model under the assumption that translational and rotational temperatures equilibrate for all species and that the diatomic molecules such as $\mathrm{O}_{2}, \mathrm{~N}_{2}, \mathrm{O}$, and NO have their own vibrational temperature. The electron-electronic temperature is also considered separately. 


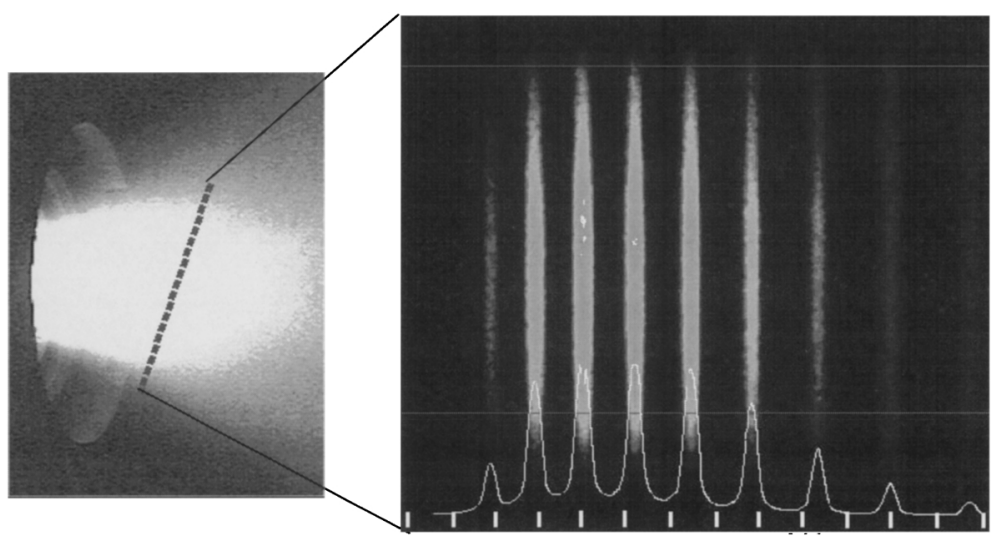

Fig. 12. Spacial distribution of the NO-LIF spectrum excited through $(v, v)=\gamma(0,0) Q_{22}(11.5)$.

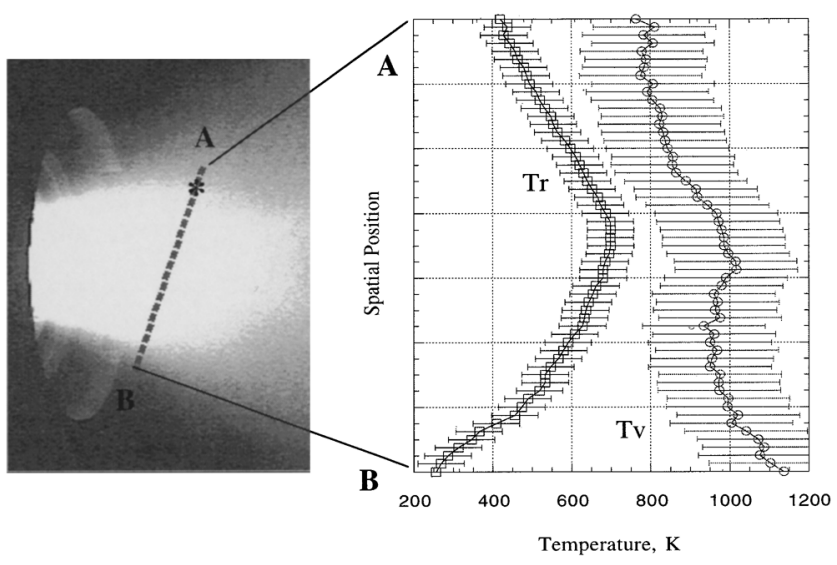

Fig. 13. Nonequilibrium temperature distribution.

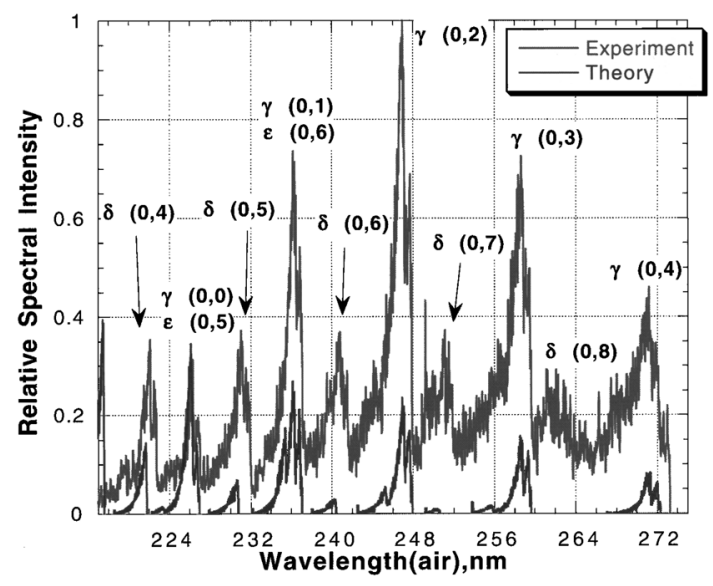

Fig. 14. Emission spectroscopic data (NO).

Many possibilities arise in the choice of chemical, physical, or microscopic models and variables that determine the nonequilibrium characteristics of the flow. Here adapted are Lee's electron-vibrational coupling model ${ }^{14)}$ and Wray's vibrational relaxation model. ${ }^{15)}$

\subsubsection{Axial distribution of the nonequilibrium temper- ature}

Figure 15 shows the axial distribution of rotational and vibrational temperature along the nozzle center-axis obtained by NO LIF in comparison with the quasi one-dimensional numerical analysis and the rotational temperature obtained by emission spectroscopy. Since the laser light is inserted perpendicular to the center-axis, the measured points are discretely at the area ratio of 16 and 100, respectively. An estimation of rotational temperature from spectroscopic data depends on the fitting range because unexpected emission bands are included in it, as previously described. The three lines estimated with different fitting ranges from the same data are also plotted in Fig. 15. At the area ratio around 100 , the nonequilibrium temperatures based on different data (LIF, emission spectroscopy, numerical analysis) correspond with one another. While in the low area ratio region around 10 , the relaxation of the emission spectroscopy rotational temperature is relatively slow, especially in comparison with the numerical analysis and the LIF data within their error bars.

The rotational temperature is one of the indexes that expresses rotational population distribution. The difference of rotational temperatures between LIF data and emission spectroscopic data designates the difference of the rotational pop-

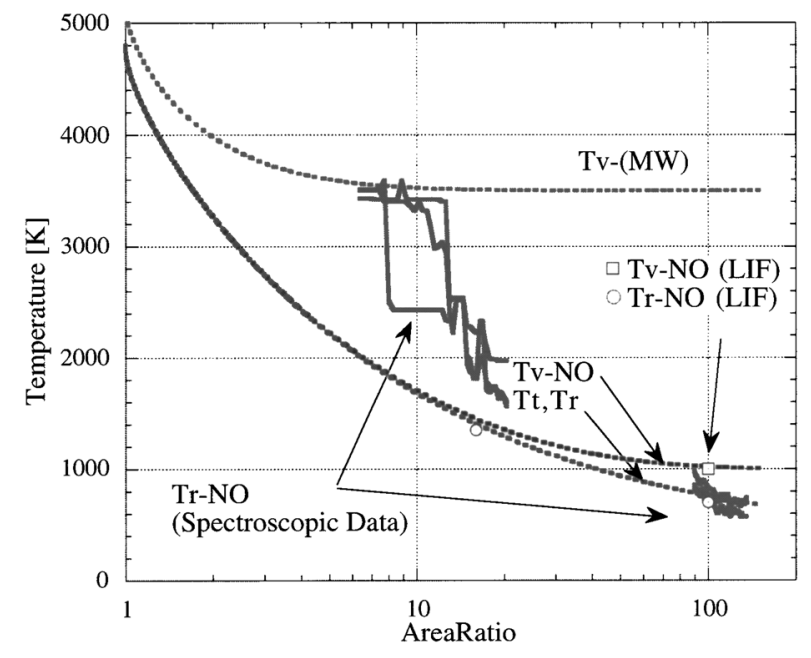

Fig. 15. Nonequilibrium temperature distribution along the nozzle center axis obtained by NO-LIF in comparison with emission spectroscopic data and the quasi 1-D numerical analysis. 
ulation distribution in the electronic state $X^{2} \Pi_{1 / 2}$ and $A^{2} \Sigma$, respectively. This result seems to show that the rotational temperature relaxation in $A^{2} \Sigma$ is slower than in $X^{2} \Pi_{1 / 2}$. Although further discussion in detail has little meaning because of insufficient data, it is possible that each electronic state has its own rotational distribution. The concept of rotational temperature itself should be carefully treated by considering whether it is a specific value for one electronic state or an averaged value among all the electronic population in the expanding arc-heated airflow. The NO-LIF thermometry of $A^{2} \Sigma$ is one of the further interesting issues.

\section{Conclusion}

Understanding of the aerothermophysical features of the high-enthalpy flow in the arc-heating facility is important for designing atmospheric hypersonic flight vehicles based on the heating test result.

Reconsiderting the effect of rotational transfer, we show in the present study a methodology of LIF thermometry and established the system of NO LIF thermometry for the measurement of arc-heated airflow. Nonequilibrium temperatures of NO $\gamma$-band $\left(A^{2} \Sigma-X^{2} \Pi_{1 / 2}\right)$ measured experimentally by 2 -line and multiline laser-induced fluorescence spectroscopy technique exhibits a separation between vibrational and rotational temperatures. When an arc-heater is operated at the enthalpy level of $10 \mathrm{MJ} / \mathrm{kg}$, the measured vibrational temperature at area ratio of 100 is about $1,000 \mathrm{~K}$, which is higher than the rotational temperature by $300 \mathrm{~K}$.

The temperature relaxation along the flow has been compared with the emission spectroscopic data. In the comparison between LIF data and spectroscopic data, the rotational temperatures show consistency in the higher area ratio region, but it shows not a small discrepancy around the region of area ratio 10. This shows that the rotational population in different electronic states differs each other.

\section{Acknowledgment}

The authors are indebted to our graduate students in those days,
M. Eng. Tetsu Takahashi, and M. Eng. Kenji Itagaki, for their outstanding assistance in conducting LIF and spectroscopy experiment.

\section{References}

1) Institute of Space and Astronautical Science: MUSES-C Mission Planning, March, 2001.

2) Yamada, T. and Inatani, Y.: ISAS High Enthalpy Flow Facility for Thermal Protection Material Tests, IEPC-99-233, 26th IEPC, Kitakyushu, Japan, Oct. 17-21, 1999.

3) Millot, G., Lavorel, B., Fanjoux, G. and Wenger, C.: Determination of Temperature by Stimulated Raman Scattering of Molecular Nitrogen, Oxygen, and Carbon Dioxide, Appl. Phys. B, 56 (1993), pp. 287-293.

4) Laufer, G., Mckenzie, R. L. and Fletcher, D. G.: Method for Measuring Temperatures and Densities in Hypersonic Wind Tunnel Air Flows Using Laser-Induced $\mathrm{O}_{2}$ Fluorescence, Appl. Opt., 29 (1990), pp. 4873-4883.

5) Pezé, P. and Siffre: Laser-induced Fluorescence Spectroscopy of The (0.0) Schumann-Runge band of $\mathrm{O}_{2}$, Opt. Lett., 19 (1994), pp. 20212023.

6) McKenzie, R. L. and Gross, K. P.: Two-Photon Excitation of Nitric Oxide Fluorescence as a Temperature Indicator in Unsteady Gasdynamic Processes, Appl. Opt., 20 (1981), pp. 2153-2165.

7) Fletcher, D. G.: Arcjet Flow Property Determined from LIF of Atomic Nitrogen, AIAA Paper 98-0205, 1998.

8) Feigl, M. and Auweter-Kurtz, M.: Investigation of Nitric Oxide in a High Enthalpy Air Plasma Flow Using LIF, AIAA Paper 98-2459, 1998.

9) Vecchio, A. D., Palumbo, G., Koch, U. and Gülhan A.: RotoTranslational Temperature Measurements of NO Molecules and $\mathrm{O}$ Atoms by 2D-LIF Spectroscopy in a Non-Equilibrium High Enthalpy Flow, AIAA Paper 99-3598, 1999.

10) Herzberg, G.: Molecular Spectra and Molecular Structure, Vol. IV, Constants of Diatomic Molecules, Van Nostrand Reinhold Co., New York, 1979.

11) Echbreth, A. C.: Laser Diagnostics for Combustion Temperature and Species, Abacus Press, Cambridge, USA, 1984.

12) Fujita, K. and Abe, T.: SPRADIAN; Structured Package of Radiation Analysis Code, ISAS Report, Vol. 669, 1997.

13) Gopaul, N. K. J. M.: Spectral Measurement of Nonequilibrium ArcJet Free Stream Flow, 39th International Instrumentation Symp., Albuquerque, NM, May 2-6, 1993, pp. 1067-1085.

14) Lee, J. H.: Electron-Impact Vibrational Relaxation in HighTemperature Nitrogen, J. Thermophys. Heat Transfer, 7, 3 (1993), pp. 399-405.

15) Wray, K. L.: Shock Tube Study of the Vibrational Relaxation of Nitric Oxide, J. Chemical Physics, 139 (1962), pp. 2597-2603. 